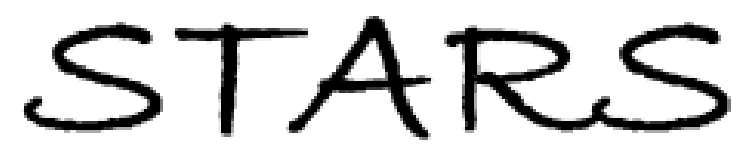

University of Central Florida

STARS

$12-31-2007$

\title{
Improved aperture for modulation transfer function measurement of detector arrays beyond the Nyquist frequency
}

Alfred D. Ducharme

Sarah P. Temple

University of Central Florida

Find similar works at: https://stars.library.ucf.edu/facultybib2000

University of Central Florida Libraries http://library.ucf.edu

This Article is brought to you for free and open access by the Faculty Bibliography at STARS. It has been accepted for inclusion in Faculty Bibliography 2000 s by an authorized administrator of STARS. For more information, please contact STARS@ucf.edu.

\section{Recommended Citation}

Ducharme, Alfred D. and Temple, Sarah P., "Improved aperture for modulation transfer function measurement of detector arrays beyond the Nyquist frequency" (2007). Faculty Bibliography 2000s. 293. https://stars.library.ucf.edu/facultybib2000/293

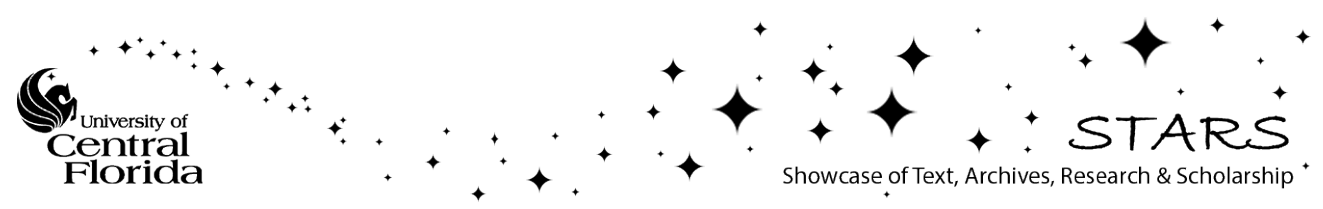




\section{Improved aperture for modulation transfer function measurement of detector arrays beyond the Nyquist frequency}

\author{
Alfred D. Ducharme \\ Sarah P. Temple \\ University of Central Florida \\ Department of Engineering Technology \\ 4000 Central Florida Blvd. \\ Orlando, Florida 32816 \\ E-mail: ducharme@mail.ucf.edu
}

\begin{abstract}
The design of an aperture for the generation of laser speckle with a flat power spectrum covering a wide band of the measurement spatial-frequency range is presented. This aperture allows for the measurement of modulation transfer function (MTF) from zero to twice the Nyquist frequency of a two-dimensional detector array. This design mitigates many of the measurement problems inherent in other aperture designs. The MTF measurement of a CCD detector array is used to demonstrate the measurement technique and illustrate the advantages of the new aperture design. (0) 2008 Society of Photo-Optical Instrumentation Engineers. [DOI: 10.1117/1.2976798]
\end{abstract}

Subject terms: modulation transfer functions; speckle; charge-coupled devices.

Paper 080040R received Jan. 17, 2008; revised manuscript received Jun. 27, 2008; accepted for publication Jul. 1, 2008; published online Sep. 5, 2008.

\section{Introduction}

The modulation transfer function (MTF) is a single plot that quantifies the ability of an imaging system to resolve features in an object with a particular size. The spatial size of these objects can also be expressed in terms of spatial frequency by inverting the period of idealized periodic objects. The MTF plot is generated by measuring the attenuation, called the modulation depth, at a series of specific spatial frequencies. The number of data points on the MTF curve is governed by the measurement technique used.

In today's imaging systems, optical lenses and mirrors produce an image on an electronic detector array. This requires the extension of traditional MTF theory for optical systems to modern electro-optical systems. The electrooptical MTF includes the performance of the detector technology as well as the electronic processing and storage of the imagery. Testing on complete systems incorporating an optical imaging system and a detector array must employ MTF measurements utilizing objects with known spatialfrequency content. Techniques commonly used are bar and sinusoidal targets [bar targets actually yield the contrast transfer function (CTF)] as well as knife-edge tests. However, these tests require imaging optics to generate the image of these targets on the detector array. There is often a need to measure the MTF of the detector array alone without the need for intervening optics. In these cases, the use of laser speckle is applicable because the speckle is projected directly onto the array. ${ }^{1,2}$

The use of laser speckle as a test input has several advantages over other MTF measurement techniques. The first is that even if a well-corrected imaging system is used to project a target such as a knife-edge onto the array, the optics are still part of the measurement. Any unwanted information introduced by the instrument must be extracted from the final measurement. It is advisable to avoid these

0091-3286/2008/\$25.00 @ 2008 SPIE types of postprocessing techniques, since they can decrease the accuracy and repeatability of the measurement. The second advantage is that laser-speckle testing measures the entire array rather than a limited area, thereby more accurately measuring the overall performance of the array. In cases where on- and off-axis MTF measurement is desired, regions of the test image can be used. Finally, a lesserknown advantage of the laser-speckle technique is that it includes the MTF due to the sampling of the image. When discrete imaging sites are used to sample an image (i.e., the pixels of the detector array), an additional MTF is incorporated into the detector MTF, called the sampling MTF. When deterministic targets- those with periodic variation of contrast - are used, they must be optimally aligned with the rows and columns of the detector array, referred to as phasing. The result of perfect alignment is that the effects of sampling are negated and the sampling MTF is equal to 1 at all frequencies. This leads to a discrepancy between laboratory measurements of MTF and the field performance of the system.

The limiting MTF of an optical imaging system utilizing a detector array is determined by the physical dimensions of a single detector (footprint) and by the sampling interval of the readout electronics. The footprint of a single detector, $\Delta x$, yields an $\mathrm{MTF}_{\text {footprint }}$ equal to $\operatorname{sinc}(\xi \Delta x)$ [i.e., $\sin (\xi \Delta x) /(\xi \Delta x)$, where $\xi$ is the spatial frequency in the $x$ dimension]. Similarly, the sampling interval, $\Delta s$, has an associated $\operatorname{MTF}_{\text {sampling }}$ equal to $\operatorname{sinc}(\xi \Delta s)$. The average limiting MTF of the system is the product of these two MTFs. For a detector array with contiguous detectors, such as a CCD array, we have $\Delta x=\Delta s$, which yields an MTF equal to $\operatorname{sinc}^{2}(\xi \Delta x){ }^{4}$

The test procedure for deterministic targets, such as bar targets, requires alignment of the target with the rows and columns of the detector array. This has the effect of forcing $\mathrm{MTF}_{\text {sampling }}$ to be equal to 1 for all frequencies. When deterministic targets with high-contrast features, such as bar 
targets, are used to test the MTF, the precise alignment

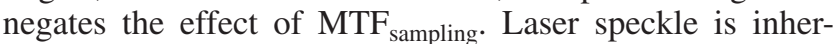
ently random, and when it is used to test the MTF, the random placement of the individual speckles and the averaging used in processing results in a MTF that includes the sampling MTF. Therefore, laser-speckle MTF measurements provide a more accurate prediction of the imaging system performance in the field. ${ }^{1}$

To date, only a limited amount of work has been done on the development of laser speckle MTF testing methods. ${ }^{1,5-9}$ Earlier work employed a two-slit aperture to produce narrowband laser speckle. ${ }^{1}$ The narrowband technique depended on a movable frequency component to test the MTF at specific spatial frequencies. The feature movement was controlled by the movement of the detector array under test with respect to the two-slit aperture. The novelty of this method was that it allowed the MTF to be measured from zero to twice the Nyquist frequency of the array. Unfortunately, this technique suffered from two major drawbacks. First, the detector array had to be moved to a new location for each spatial frequency that needed to be measured. This required precision movement along an optical stage, often motorized and computer-controlled. Second, each measurement needed to be normalized against lowfrequency measurements. This normalization is difficult in practice and yields low repeatability between measurements. Other researchers have made attempts to mitigate this problem, but ultimately the measurement complexity is increased. ${ }^{6,9}$

In this paper, we present a radically new aperture design for generation of wideband laser speckle. This new aperture yields a flat power spectrum from near-zero spatial frequency out to a cutoff frequency defined by a physical distance in the measurement setup. The aperture is used to collect data at two distances determined by the detector array being tested. The first distance spreads the flat input power spectrum from zero to the Nyquist frequency of the array. The data collected are processed to yield the continuous MTF from zero to the Nyquist frequency. The second distance is half the first and spreads the flat input spectrum from zero to twice the Nyquist frequency of the array. A simple division-and-unfolding operation with the first measured spectrum yields the MTF from the Nyquist frequency to twice the Nyquist frequency of the array. The two MTF measurements are then combined to yield a complete measurement of the array from zero to twice the Nyquist frequency. The improvement provided with this aperture is a reduction in movement and more accurate normalization of the data.

In Sec. 2 we present the theoretical development of the new aperture design. In Sec. 3, we demonstrate the use of this aperture using a commercially available chargecoupled device (CCD) detector array. Finally, we summarize the advantages of this new design.

\section{Cross-Aperture Design}

The use of laser speckle to measure the MTF of a detector array is based on a well-demonstrated relationship between the second-order statistic-the power spectral density (PSD)_of the laser speckle spatial distribution and the MTF of any imaging system. This relationship is expressed as
$\operatorname{PSD}_{\text {out }}(\xi, \eta)=\operatorname{PSD}_{\text {in }}(\xi, \eta)[\operatorname{MTF}(\xi, \eta)]^{2}$,

where $\xi$ and $\eta$ are the spatial frequencies in the $x$ and $y$ directions. ${ }^{10}$ Based on this relationship, the objective of a laser speckle generator is to provide the measurement with a $\mathrm{PSD}_{\text {in }}$ that contains a useful distribution of features. As an example of the term useful, a $\mathrm{PSD}_{\text {in }}$ with a single triangular feature would allow the measurement of the MTF at the peak of the feature. The goal is to create a known $\mathrm{PSD}_{\text {in }}$ so that the MTF can be found through simple division as described in Eq. (1).

The laser speckle generator consists of a coherent light source that is spatially randomized and then directed through an aperture with a specific geometry. The physical shape of the aperture governs the resulting PSD of the laser speckle. Following Goodman, we observe that the PSD of the laser speckle pattern consists of a $\delta$-function component at zero frequency plus a component extended over a frequency band and having the shape of the normalized autocorrelation function of the intensity distribution existing at the output of the aperture. ${ }^{11}$ Therefore, the PSD of the laser speckle pattern can be predicted easily, using an autocorrelation algorithm in a computer. This approach was utilized to evaluate the viability of many different aperture geometries before arriving at the one proposed in this paper.

The primary disadvantages of previously researched aperture designs are not evident when they are demonstrated in a laboratory environment. When these designs are employed in industrial settings where the test is repeated on many different arrays, several problems arise. One of the most common designs, the two-slit aperture, has been demonstrated to be highly effective, and several measurements have been published. However, this aperture requires either precision positioning or careful software processing. Both are prone to processing errors, and repeatability is difficult to achieve in an automated instrument. These errors are produced by a combination of two problems.

The first problem is that the test frequency must either be calculated from the distance between the aperture and the detector array or from a software centroiding algorithm. The distance is not always easily obtainable, as a result of detector enclosures and array cover plates. The centroiding algorithm works well at low frequencies when the outer frequency component in the power spectrum is compact and defined. At high frequencies the outer frequency component spreads out and becomes less defined. The result is increasing error with increasing frequency.

The second problem is in the normalization between power specta. The two-slit aperture requires a unique data image to be collected at each discrete measurement distance (corresponding to each spatial frequency measured). The final step in processing each image and subsequent power spectrum is to normalize it to a relative power determined from the low-frequency component. This normalization process is dependable for high-spatial-frequency measurement distances, but becomes less so as the measurement frequency decreases. As the frequency decreases the low-frequency component spans a smaller range of frequencies and in effect compresses the features. The amplitude of the low-frequency component is used as the basis of the normalization. At low measurement frequencies the zero-frequency $(\mathrm{dc})$ bias in the data image blends with the 


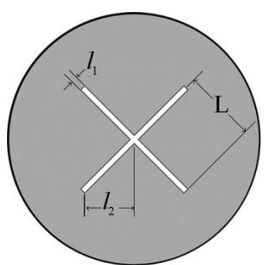

(a)

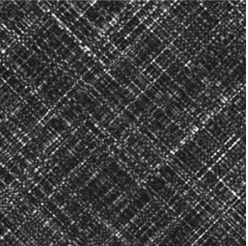

(b)
Fig. 1 Cross-aperture design: (a) physical dimensions and (b) example laser speckle pattern.

low-frequency component, making it difficult to determine the true amplitude for use in the normalization. The result is an increasing error with decreasing frequency.

These two problems have the combined effect of generating errors over the entire frequency range with the smallest error at midband frequencies. The distance measurement problem produces errors at high frequency, and the normalization problem produces errors at low frequency. These errors can be reduced with careful manual data collection. This can be achieved in a laboratory but is nearly impossible to achieve in a highly automated measurement instrument such as would be used in an industrial setting.

An improved aperture design would be one that reduces software normalization uncertainties and precision motion. The design we propose achieves these improvements by generating a flat spectrum over a wide band of spatial frequencies. The flat spectrum simplifies the determination of the low-frequency normalization value so that it can be automated easily. It also reduces the need to move the aperture for the collection of image data, since a range of measurements can be obtained at a single distance.

Our aperture design, shown in Fig. 1, is referred to as the cross aperture. It consists of an equal pair of narrow slits rotated by 45 degrees from the vertical axis with dimensions $l_{1}, l_{2}$, and $\mathrm{L}$ as shown.

The raw laser speckle pattern shown in Fig. 1(b) was generated using a computer simulation of the laser speckle optical phenomenon. The resulting two-dimensional power spectrum of this laser speckle pattern is shown in Fig. 2(a),

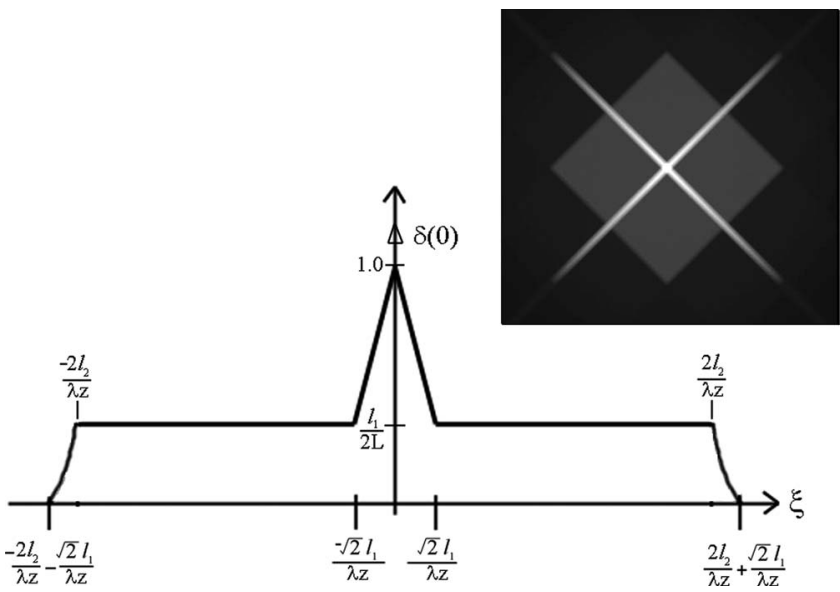

Fig. 2 Power spectral density of laser speckle generated using cross aperture: (a) two-dimensional distribution and (b) cross section through horizontal dimension. and the horizontal cross section through the middle of the pattern is given in Fig. 2(b). The PSD of the cross aperture was determined through numerical calculation.

The spatial frequency of any particular feature in the power spectrum scales as follows:

$\xi=\frac{x}{\lambda z}$

where $x$ is the corresponding aperture dimension, $\lambda$ is the wavelength of the coherent light source, and $z$ is the distance from the aperture to the observation plane. Using Eq. (2) and the dimensions of the aperture, the cutoff frequency of the wideband cross-aperture power spectrum is found to be

$\xi_{\text {cutoff }}=\frac{2 l_{2}}{\lambda z}$

Observe that the power spectrum falls off parabolically from the cutoff frequency to a value of zero. This is a result of the square end shape of the slits.

The height of the wide-band portion of the power spectrum is equal to half the ratio of the slit width $l_{1}$ to the slit length $L$. The absolute height is inconsequential since the power spectrum is normalized to the value measured at

$\xi=\frac{\sqrt{2} l_{1}}{\lambda z}$.

This normalization is demonstrated in the next section.

This aperture can be used to measure the entire MTF of a detector array from zero to twice the Nyquist frequency of the array, using a difference-and-unfolding process. Before detailing this process it is imperative that the concept of aliasing be understood.

The Nyquist frequency is half the sampling frequency of a discrete signal-processing system. In an electro-optic imaging system, the Nyquist frequency is equal to $1 /(2 \Delta x)$, where $\Delta x$ is the pixel width. In sampling theory optimal performance is achieved when the signal bandwidth is just lower than the Nyquist frequency. This is not the case in an electro-optic imaging system. As a result, a sampling artifact called aliasing is generated. Aliasing is the folding of spatial content above the Nyquist frequency of the array into lower spatial frequencies. For this reason the Nyquist frequency is sometimes referred to as the "folding frequency." The only way that aliasing can be avoided is to employ an antialiasing filter before the detector samples the image. The objective of the laser-speckle MTF measurement is to measure the complete performance of a bare detector. Therefore, the aliasing effect must be considered carefully.

To measure the complete MTF with the cross aperture, the measurement process requires that two separate sets of data be collected. The first set, called the Nyquist set, is collected at a distance that spreads the flat input $\mathrm{PSD}_{\text {in }}$ up to a cutoff frequency equal to the Nyquist frequency. The second set, called the twice-Nyquist set, is collected at a distance that spreads the flat input $\mathrm{PSD}_{\text {in }}$ beyond the $\mathrm{Ny}$ quist frequency to twice the Nyquist frequency. Higher accuracy can be achieved from the measurement if a series of 


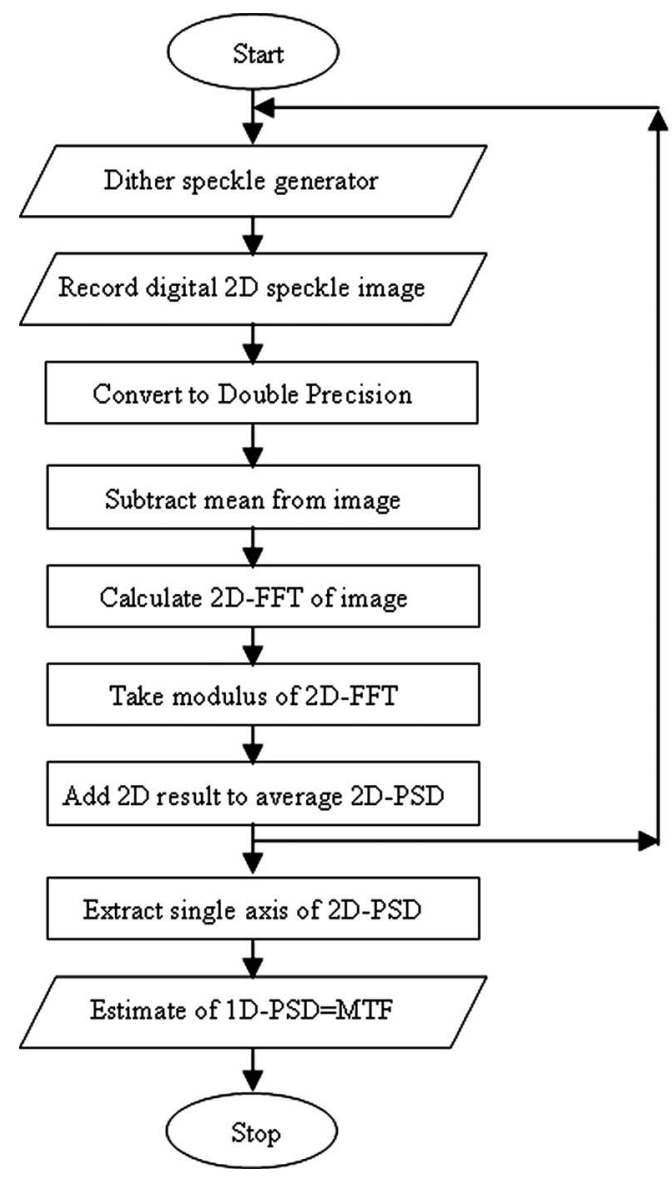

Fig. 3 PSD processing algorithm. MTF is for associated axis extracted from 2-D PSD.

data images are collected at each of the two distances.

Each image in the Nyquist set is processed using a twodimensional Fourier transformation algorithm to yield the $\mathrm{PSD}_{\text {out }}$ for the laser speckle pattern. An estimate $\mathrm{PSD}_{1 \mathrm{Ny} \text {,out }}$ is generated by averaging the processed collection of power spectra. The same processing is applied to the twiceNyquist set to yield $\mathrm{PSD}_{2 \mathrm{Ny} \text {,out }}$. The PSD processing algorithm is described in Fig. 3.

At this point, the Nyquist set has produced $\mathrm{PSD}_{1 \mathrm{Ny}, \text { out }}$ from zero to the Nyquist frequency of the array, which is the first half of the overal power spectrum, referred to as $\mathrm{PSD}_{0-\mathrm{Ny}}$. However, the result from the twice-Nyquist set, $\mathrm{PSD}_{2 \mathrm{Ny}, \text { out }}$, contains the sum of the low- and highfrequency spectra as a result of aliasing. The power spectrum $\mathrm{PSD}_{2 \mathrm{Ny}-\mathrm{Ny}}$ from Nyquist to twice-Nyquist is obtained by subtraction,

$\operatorname{PSD}_{2 \mathrm{Ny}-\mathrm{Ny}}(\xi)=\operatorname{PSD}_{2 \mathrm{Ny}, \text { out }}(\xi)-\operatorname{PSD}_{0-\mathrm{Ny}}(\xi)$,

performed along a single dimension, $\xi$. Observe that the subscript $2 \mathrm{Ny}-\mathrm{Ny}$ indicates that the data are in reverse order, from highest to lowest. The data need to be unfolded to reverse this order to $\mathrm{Ny}-2 \mathrm{Ny}$ before they represent the second half of the overall PSD.

The power spectrum $\mathrm{PSD}_{2 \mathrm{Ny}-\mathrm{Ny}}$ is unfolded using the following expression:

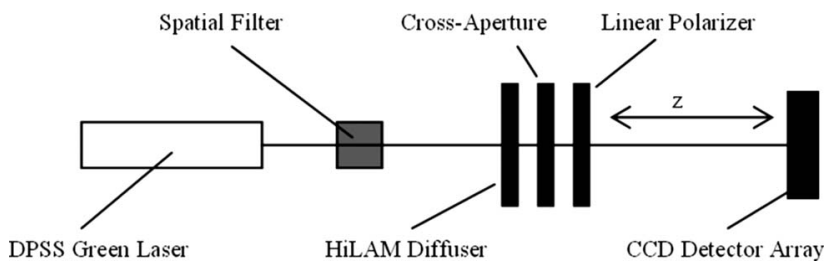

Fig. 4 Experimental setup used to demonstrate the cross aperture.

$\operatorname{PSD}_{\mathrm{Ny}-2 \mathrm{Ny}}(\xi)=\operatorname{PSD}_{2 \mathrm{Ny}-\mathrm{Ny}}\left(2 \xi_{\mathrm{Ny}}-\xi\right)$ for $\xi=\xi_{\mathrm{Ny}}$ to $2 \xi_{\mathrm{Ny}}$.

The final $\mathrm{PSD}_{\text {out,final }}$ is the combination of $\mathrm{PSD}_{0-\mathrm{Ny}}$ and $\mathrm{PSD}_{\mathrm{Ny}-2 \mathrm{Ny}}$ :

$\operatorname{PSD}_{\text {out,final }}(\xi)=\left\{\begin{array}{ll}\operatorname{PSD}_{0-\mathrm{Ny}}(\xi), & 0<\xi<\xi_{\mathrm{Ny}}, \\ \operatorname{PSD}_{\mathrm{Ny}-2 \mathrm{Ny}}(\xi), & \xi_{\mathrm{Ny}}<\xi<2 \xi_{\mathrm{Ny}}\end{array}\right.$.

Since $\mathrm{PSD}_{\text {in }}$ is uniform over the measurement range, it can be considered to be equal to 1 . The MTF of the detector array is then calculated using Eq. (1).

\section{Demonstration of Cross-Aperture MTF Measurement}

An MTF measurement of a commercially available CCD detector array was completed to demonstrate the applicability of the cross aperture. The experimental setup used to collect the laser speckle data is here described, with an explanation of the data processing.

The experimental setup used for our measurement is illustrated in Fig. 4. A diode-pumped solid-state (DPSS) laser operating at $532 \mathrm{~nm}$ was used as the coherent source. The spatial filter expanded the beam incident on the diffuserand-aperture combination. The HiLAM diffuser is a microlens high-efficiency diffuser and was previously demonstrated to be an excellent randomizer for the generation of laser speckle. ${ }^{11,12}$ The spacing between the HiLAM diffuser and the cross aperture was $12 \mathrm{~mm}$. The output of the cross aperture is passed through a linear polarizer to limit the output to a single laser speckle pattern. The distance $z$ is actually measured from the cross-aperture plane to the detector plane. This distance is used to set the cutoff frequency of the input PSD.

The dimensions of the cross aperture used for this experiment were $l_{1}=500 \mu \mathrm{m}, l_{2}=3.54 \mathrm{~mm}$, and $L=5 \mathrm{~mm}$. These dimensions give a 1:20 ratio between the heights of the low-frequency component and the wideband component in the PSD as shown in Fig. 2(b). The aperture was lasercut in $13-\mu \mathrm{m}$ stainless steel and mounted in a 25-mm-diameter aperture holder ring.

The detector array utilized was an Imaging Source DMK31BF03 monochrome CCD FireWire camera. The 1/3-in. format array has a resolution of 1024 by 768 pixels with square $4.65-\mu \mathrm{m}$ pixels. The camera was controlled and images were collected using the supplied IC Capture software. Once the exposure and gain were adjusted to yield an unsaturated histogram, their values were fixed. All noise reduction and sharpness settings were disabled.

The Nyquist frequency for a CCD with contiguous pixels is equal to $1 /(2 \Delta x)$, where $\Delta x$ is the pixel width of 


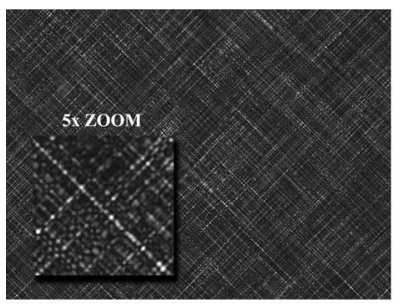

(a)

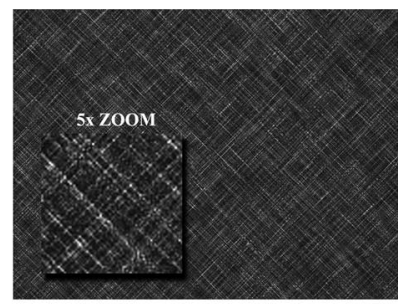

(b)
Fig. 5 Example raw speckle patterns for the (a) Nyquist set and (b) twice-Nyquist set. Both are shown with five times zoom inset.

$4.65 \mu \mathrm{m}$. Using this relationship, the Nyquist frequency for the DMK31BF03 is 107.53 cycles/mm. The required distance $z$ to set the cutoff frequency of the $\mathrm{PSD}_{\text {in }}$ at this Nyquist frequency is calculated by solving for $z$ in Eq. (3) and is equal to $123.6 \mathrm{~mm}$. The required distance $z$ to set the cutoff frequency of $\mathrm{PSD}_{\text {in }}$ at twice the Nyquist frequency is equal to $61.8 \mathrm{~mm}$.

A total of 100 images $(N=100$ in Fig. 3$)$ were collected at each of the two calculated distances, 123.6 and $61.8 \mathrm{~mm}$. A unique speckle pattern was generated for each image by making small displacements or dithering the HiLAM diffuser. This was done so that an averaging process could be used to increase the accuracy of the final MTF. Without averaging the MTF produced would be dominated by noise. Since the images are composed of random features, the 2-D PSD will also contain random features. As a result, increased accuracy is obtained by averaging the 2-D PSDs to form a better estimate of the PSD and consequently a more accurate measurement of the MTF. Example speckle images at each distance are shown in Fig. 5.

The Nyquist cut-off data set was processed using a 2-D Fourier transformation algorithm to yield an estimate of $\mathrm{PSD}_{\text {out }}$. The measurement was then normalized to yield the MTF of the DMK31BF03 camera from near-zero spatial frequency to the Nyquist frequency. This result is shown in Fig. 6.

Data from zero to approximately 10 cycles $/ \mathrm{mm}$ have been discarded, since they correspond to the low-frequency triangular feature in the PSD as shown in Fig. 2. The data were normalized to the value just after this triangular feature at a spatial frequency of $\xi=\left(\sqrt{2} l_{1}\right) /(\lambda z)$ $=10.75$ cycles $/ \mathrm{mm}$.

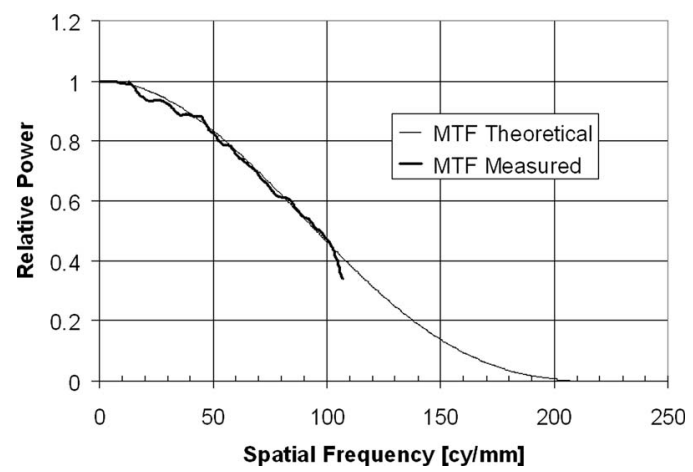

Fig. 6 Measured MTF of DBK31BF03 camera for Nyquist cutoff image data.

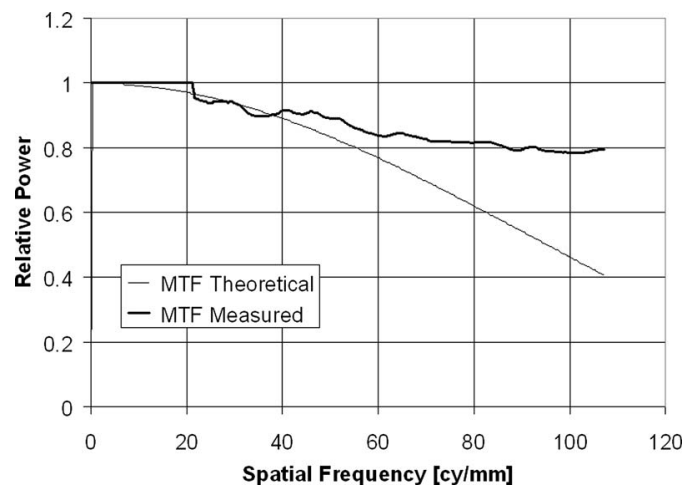

Fig. 7 Measured MTF of DBK31BF03 camera for twice-Nyquist cutoff image data, shown before division and unfolding operations.

The introduction of spatial-frequency information above the Nyquist frequency of the array causes a folding of the power into lower spatial frequencies. Content at a spatial frequency of $\xi_{\text {Nyquist }}+\xi_{i}$ collected by the array will be realized at a spatial frequency equal to $\xi_{\text {Nyquist }}-\xi_{i}$. The second set of image data, collected at twice the Nyquist frequency, will contain information from Nyquist to twice-Nyquist added to the information from zero to Nyquist. In order to separate the data, a division between the Nyquist MTF estimate and the twice-Nyquist one must be performed. In addition, the result must have its order reversed before it is plotted as the second half of the final MTF curve. This is referred to as the unfolding operation.

The twice-Nyquist set of data was processed using the same algorithm as the Nyquist set. The resulting data are shown in Fig. 7 before division and unfolding. Once again, data from approximately zero to 21 cycles/mm have been discarded. The data were normalized to the value just after the triangular feature at a spatial frequency of $\xi$ $=\left(\sqrt{2} l_{1}\right) /(\lambda z)=21.5$ cycles $/ \mathrm{mm}$. The measured MTF shown in Fig. 7 shows that there is additional information contained in the result. This causes the measured curve to have values well above the theoretical curve shown. The measured MTF curves shown in Figs. 6 and 7 are then divided and unfolded to yield the final MTF of the array, shown in Fig. 8.

The discontinuity at the Nyquist frequency is a result of the lack of data near there. It is possible to reduce this error

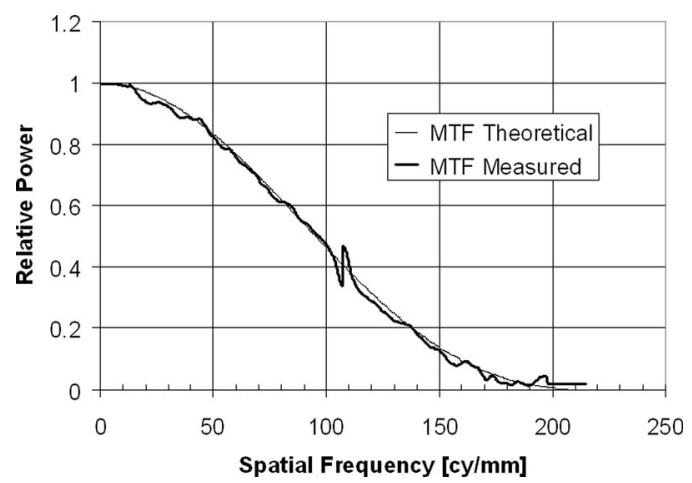

Fig. 8 Final MTF measurement on DBK31BF03 camera over entire response of camera for the horizontal dimension. 


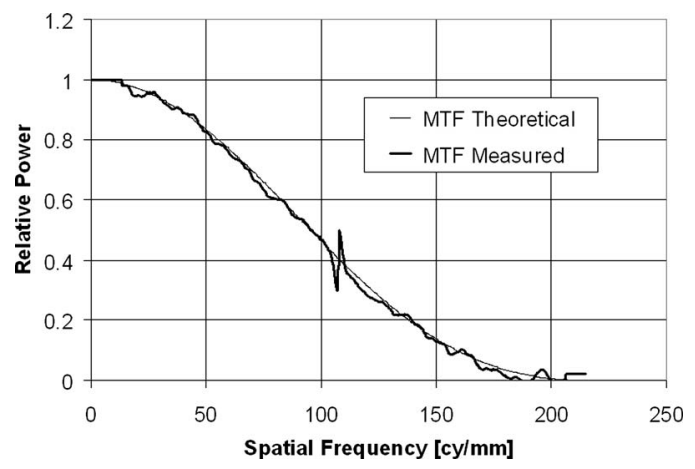

Fig. 9 Final MTF measurement on DBK31BF03 camera over entire response of camera for the vertical dimension.

with increased data collection and more precise distance alignment of the array. The noise seen at high frequencies is expected, since the measurement is nearing the noise floor of the camera.

The MTF measurement shown in Fig. 8 includes 1024 data points, since the MTF along the horizontal axis of the $\mathrm{CCD}$ is measured. The MTF along the vertical axis can also be obtained using the same data collected and yields 768 data points. This additional measurement is shown in Fig. 9 .

\section{Summary}

The cross-aperture design has been demonstrated as an improved alternative to earlier designs such as the two-slit aperture. This improved design generates a uniform frequency distribution extended to a deterministic cutoff frequency. The cutoff frequency is set by the distance from the cross aperture to the observation plane. A collection of laser speckle images is obtained at two distances to measure the complete MTF of a detector array from zero to twice the Nyquist frequency. The data-processing technique called unfolding was demonstrated on a CCD array, and its results compared with the theoretical MTF of an ideal CCD. The close match between the measured and theoretical MTFs is presented as proof that this technique is viable.

\section{References}

1. M. Sensiper, G. D. Boreman, A. D. Ducharme, and D. Snyder, "Modulation transfer function testing of detector arrays using narrowband laser speckle," Opt. Eng. 32(2), 395-400 (1993).
2. G. D. Boreman, Y. Sun, and A. James, "Generation of laser speckle with an integrating sphere," Opt. Eng. 29(4), 339-342 (1990).

3. G. D. Boreman and A. E. Plogstedt, "Spatial filtering by a linescanned non-rectangular detector: application to SPRITE readout MTF," Appl. Opt. 28, 1165-1168 (1989).

4. E. L. Dereniak and G. D. Boreman, Infrared Detectors and Systems, 1st ed., Vol. 1, Chap. 13, p. 514, John Wiley \& Sons, Inc., New York (1996).

5. G. D. Boreman and E. L. Dereniak, "Method for measuring modulation transfer function of charge-coupled devices using laser speckle," Opt. Eng. 25(1), 148-150 (1986).

6. W. Astar, "New power-efficient optical filter for detector array modulation transfer function measurement by laser speckle," Opt. Eng. 35(9), 2761-2679 (1996).

7. A. M. Pozo and M. Rubiño, "Optical characterization of ophthalmic lenses by means of modulation transfer function determination from a laser speckle pattern," Appl. Opt. 44, 7744-7748 (2005).

8. A. M. Pozo and M. Rubiño, "Comparative analysis of techniques for measuring the modulation transfer functions of charge-coupled devices based on the generation of laser speckle," Appl. Opt. 44, 1543 -1547 (2005)

9. A. M. Pozo, A. Ferrero, M. Rubiño, J. Campos, and A. Pons, "Improvements for determining the modulation transfer function of charge-coupled devices by the speckle method," Opt. Express 14, 5928-5936 (2006)

10. L. Goldfischer, "Autocorrelation function and power spectral density of laser-produced speckle patterns," J. Opt. Soc. Am. 55, 247-252 (1965).

11. J. W. Goodman, "Statistical properties of laser speckle patterns," in Laser Speckle and Related Phenomena, 2nd ed., J. C. Dainty, Ed., Vol. 9, Chap. 2, p. 40, Springer-Verlag, Berlin (1984).

12. A. Ducharme, "Microlens diffusers for efficient laser speckle generation," Opt. Express 15, 14573-14579 (2007).

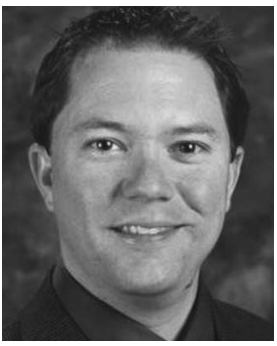

Alfred D. Ducharme is an assistant professor of engineering technology in the College of Optics and Photonics at the University of Central Florida. He is currently the assistant dean of distance and distributed learning for the College of Engineering and Computer Science. He is also the program coordinator for the BSEET photonics program instituted in 2003. His research interests include solid-state lighting, imaging system testing, and laser speckle. Dr. Ducharme earned his BS in electrical engineering from the University of Massachusetts, Lowell, and a $\mathrm{PhD}$ in electrical engineering from the University of Central Florida (CREOL). Dr. Ducharme was awarded the Rudolf Kingslake award by SPIE in 1995 and has 18 U.S. patents.

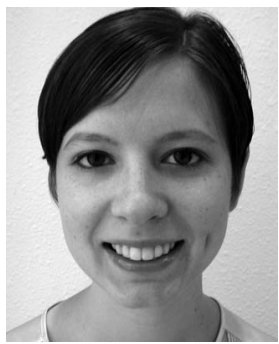

Sarah Temple has a bachelor's degree from the University of Central Florida in electrical engineering technology with a specialization in photonics. While studying at the university, her research was on modulation transfer functions. Currently, she works as a laser reliability engineer for Northrop Grumman Laser Systems. 